\title{
LOCALLY COMPACT TRANSFORMATION GROUPS AND $C^{*}$-ALGEBRAS
}

\author{
BY EDWARD G. EFFROS ${ }^{1}$ AND FRANK HAHN ${ }^{2}$ \\ Communicated by G. A. Hedlund, October 4, 1966
}

It has long been recognized that one may associate operator algebras with transformation groups (see, e.g. [9, Chapter III], $[11 ; 1$, p. 310] [5]). In this paper we shall answer two questions about the ergodic invariant probability measures on a locally compact transformation group (Theorems 2 and 3). This information is then used to solve analogous problems for the unit traces of a $C^{*}$-algebra (Theorems 5 and 6). Full proofs will appear elsewhere.

Let $(G, Z)$ be a topological transformation group with $G$ and $Z$ second countable and Hausdorff, $G$ a locally compact group, and $Z$ a compact space. Let $Z / G$ be the set of orbits $G \zeta$ with $\zeta$ in $Z$, together with the quotient topology. Define an equivalence relation $\sim$ on $Z / G$ by $p \sim q$ if the sets $\{p\}$ and $\{q\}$ have the same closure, and let $(Z / G)^{\sim}$ be the equivalence classes with the quotient topology (see $[8$, p. 58]). The elements of $(Z / G)^{\sim}$ are in one-to-one correspondence with the subsets of $Z$ that are closures of orbits. $Z / G$ is $T_{0}$ if and only if $\sim$ is trivial, and $T_{1}$ if and only if the orbits are closed.

Let $Z$ be compact and let $C(Z)$ be the continuous complex valued functions on $Z$ with the uniform norm, and $M(Z)=C_{r}(Z)^{*}$ the real Radon measures on $Z$ with the weak* topology. Let $G$ act on $C(Z)$ and $M(Z)$ by translation, i.e., for $s$ in $G, \zeta$ in $Z, f$ in $C(Z)$, and $\mu$ in $M(Z)$, let

$$
\begin{aligned}
& (s f)(\zeta)=f\left(s^{-1} \zeta\right), \\
& (s \mu)(f)=\mu\left(s^{-1} f\right) .
\end{aligned}
$$

Let $M_{G}(Z)$ be the invariant measures on $Z$, and $P_{G}(Z)$ the corresponding probability measures, i.e.,

$$
P_{G}(Z)=M_{G}^{+}(Z) \cap H,
$$

where $M_{G}^{+}(Z)$ are the positive invariant measures, and $H$ are the measures $\mu$ such that $\mu(Z)=1 . P_{G}(Z)$ is a compact simplex in the sense of Choquet, and its extremal points are just the ergodic mea-

1 This research was supported in part by the Office of Naval research (NONR $551(57)$ ).

${ }^{2}$ National Science Foundation Fellow. 
sures (see $[10, \S 10])$. Let $E P_{G}(Z)$ be the extreme points together with the simplex structure topology. A subset is defined to be closed in this topology if it consists of the extreme points of a closed face in $P_{G}(Z)$. $E P_{G}(Z)$ is compact, and it is Hausdorff if and only if $E P_{G}(Z)$ is closed in $P_{G}(Z)$ (see [4]).

The support of an ergodic measure is the closure of an orbit (see $[8$, p. 59]), hence we may define a map

$$
\theta: E P_{G}(Z) \rightarrow(Z / G) \sim
$$

by letting $\theta(\mu)$ correspond to the support of $\mu$. The following is verified:

THEOREM $1 . \theta$ is continuous. In addition

(a) If $Z / G$ is $T_{0}$, then $\theta$ is one-to-one.

(b) If the orbits are closed, $\theta$ is onto.

(c) If the orbits are finite, and uniformly bounded in cardinality, $\theta$ is a homeomorphism.

(d) If the orbits have the same finite cardinality, then $E P_{G}(Z)$ and $(Z / G) \sim$ are Hausdorff.

(e) If $G$ is equicontinuous, $\theta$ is a homeomorphism onto, and $E P_{G}(Z)$ and $(Z / G) \sim$ are Hausdorff.

In particular, if all of the orbits are finite, then $\theta$ is a continuous bijection. On the other hand our first construction shows:

THEOREM 2. There is a distal action of the integers $G$ on a compact metric space $Z$ such that all of the orbits are finite, but $\theta$ is not a homeomorphism.

For many transformation groups, $\theta$ is not one-to-one. In fact we have proved:

THEOREM 3. There is a $C^{\infty}$ distal action of the integers $G$ on the torus $Z$ such that $(Z / G)^{\sim}$ has only one point (i.e., $(G, Z)$ is minimal), and $E P_{G}(Z)$ is uncountable.

Let $\mathfrak{A}$ be a separable $C^{*}$-algebra with identity. Let $\mathrm{pr} \mathfrak{A}$ be the set of primitive ideals in $\mathfrak{A}$ with the Jacobson structure topology (see $[3, \S 3])$. Let $\mathfrak{A}^{*}$ be the Banach dual of $\mathscr{A}$ with the weak* topology. The central functions $C(\mathfrak{H})$ are the $f$ in $\mathfrak{I}^{*}$ such that $f(A B)=f(B A)$ for all $A$ and $B$ in $\mathfrak{A}$. Let $T(\mathfrak{A})$ be the unit traces on $\mathfrak{A}$, i.e.,

$$
T(\mathfrak{Q})=C^{+}(\mathfrak{R}) \cap H,
$$

where $C^{+}(\mathfrak{Q})$ are the positive central functions, and $H$ consists of the $f$ in $\mathfrak{H}^{*}$ such that $f(I)=1 . T(\mathfrak{A})$ is a compact simplex (see [12, Satz 1$]$ ), 
and its extreme points are just the traces that give rise to factor representations (see $[3, \S 6.7 .3]$ ). Let $E T(\mathfrak{A})$ be the extreme traces, with the simplex structure topology.

The kernel of a factor representation of $\mathfrak{A}$ is primitive (see [2, p. 100]). This enables us to define a map

$$
\theta^{\prime}: E T(\mathfrak{H}) \rightarrow \operatorname{pr} \mathfrak{A}
$$

by

$$
\theta^{\prime}(\tau)=\operatorname{kernel} L^{\tau},
$$

where $L^{\tau}$ is the representation defined by $\tau$.

Theorem. 4. $\theta^{\prime}$ is continuous. In addition,

(a) If $\mathfrak{A}$ is of type I, then $\theta^{\prime}$ is one-to-one.

(b) If all of the representations of $\mathfrak{A}$ are finite dimensional, then $\theta^{\prime}$ is onto.

(c) If the irreducible representations of $\mathfrak{A}$ have dimension uniformly bounded by a finite cardinal, then $\theta^{\prime}$ is a homeomorphism.

(d) If all of the irreducible representations of $\mathfrak{A}$ are of the same finite dimension, then ET(I) and pr $\mathfrak{A}$ are Hausdorff.

Letting $\mathfrak{A}(G, Z)$ be the $C^{*}$-algebra associated with a transformation group $(G, Z)$ (see [6, p. 890]) we may use Theorems 2 and 3 to prove

Theorem 5. There is a separable $C^{*}$-algebra $\mathfrak{A}$ for which all of the representations are finite dimensional, and $\theta^{\prime}$ is not a homeomorphism.

Theorem 6. There is a separable $C^{*}$-algebra $\mathfrak{A}$ such that pr $\mathfrak{A}$ has only one point (i.e., $\mathfrak{A}$ is simple), and ET( $\mathfrak{R})$ is uncountable.

Sketching the proofs of Theorems 5 and 6 , assume that $G$ is discrete and $Z$ is compact, and let $\mathfrak{A}=\mathfrak{A}(G, Z)$. Consider the diagram

$$
\begin{aligned}
E P_{G}(Z) & \stackrel{\pi^{\prime}}{\leftarrow} E T(\mathfrak{A}) \\
\downarrow \theta \quad & \quad \downarrow \theta^{\prime} \\
(Z / G) \sim & \stackrel{\pi}{\leftarrow} \text { pr } \mathfrak{A} \\
& \rightarrow
\end{aligned}
$$

$\theta$ and $\theta^{\prime}$ are defined above. $C(Z)$ may be regarded as a subalgebra of $\mathfrak{A}$, and if $P$ is a primitive ideal in $\mathfrak{A}$, there is an orbit closure $F$ in $Z$ such that

$$
P \cap C(Z)=\{f \in C(Z): f \mid F=0\} .
$$


$\pi(P)$ is defined to be the corresponding element of $(Z / G)^{\sim} . \pi$ is continuous and onto.

If $\tau$ is an extremal trace, its restriction to $C(Z)$ is an ergodic measure (see [12, Lemma 14]). Letting $\pi^{\prime}$ be the restriction map, $\pi^{\prime}$ is continuous and onto (see [12, Lemma 16]), and the diagram is commutative.

The isotropy group $H_{\zeta}$ at $\zeta$ consists of the $s$ in $G$ for which $s \zeta=\zeta$. Irreducible representations of $H_{\zeta}$ may be induced to irreducible representations of $\mathfrak{A}$ (see [6, p. 901]). Inducing the trivial one-dimensional representation at $\zeta$, one obtains a map $T_{1}$ of $Z$ into pr $\mathfrak{A}$. If the isotropy groups "vary continuously" with $\zeta$, it follows from [6, Theorem 2.1] that $T_{1}$ defines a continuous map $T$ of $(Z / G)^{\sim}$ into $\mathrm{pr} \mathfrak{A}$ which is a cross-section for $\pi$. This condition on isotropy groups is too strong for our purposes. We have been able to prove:

THEOREM 7. If the isotropy groups are commutative, then $T_{1}$ induces a continuous cross-section $T$ for $\pi$.

We have also generalized Theorem 7 to locally compact $G$ and $Z$.

Turning to Theorem 5 , let $\mathfrak{A}=\mathfrak{A}(G, Z)$, where $(G, Z)$ is described in Theorem 2. As the orbits are closed, the action of $G$ on $Z$ is smooth, and by Mackey's Imprimitivity Theorem, all of the irreducible representations of $\mathfrak{A}$ are induced from characters on isotropy groups $H_{\zeta}$ (see [6, Theorem 2.2]). As the latter are of finite index in $G$, the irreducible representations are finite dimensional. It follows from Theorems 1 and 4 that $\theta$ and $\theta^{\prime}$ are both bijections. Let $\mu_{\alpha}$ and $\mu$ be in $E P_{G}(Z)$ with $\theta\left(\mu_{\alpha}\right)$ converging to $\theta(\mu)$, but $\mu_{\alpha}$ not converging to $\mu$. We have

$$
P_{\alpha}=T\left(\theta\left(\mu_{\alpha}\right)\right) \rightarrow P=T(\theta(\mu)),
$$

hence if $\theta^{\prime}$ is a homeomorphism,

$$
\tau_{\alpha}=\theta^{\prime-1}\left(P_{\alpha}\right) \rightarrow \tau=\theta^{\prime-1}(P) .
$$

As

$$
\theta\left(\mu_{\alpha}\right)=\pi \theta^{\prime}\left(\tau_{\alpha}\right)=\theta \pi^{\prime}\left(\tau_{\alpha}\right)
$$

and $\theta$ is one-to-one, $\mu_{\alpha}=\pi^{\prime}\left(\tau_{\alpha}\right)$ and similarly, $\mu=\pi^{\prime}(\tau)$. From (1), $\mu_{\alpha} \rightarrow \mu$, a contradiction.

$(G, Z)$ is said to be free if the isotropy groups are trivial. $G$ is amenable if the regular representation of $G$ weakly contains all of the irreducible representations (see $[3, \S 18.3]$ and $[7, \S 2.3]$ ). Generalizing a result of Guichardet for semidirect products $[8$, p. 58], 
ThEOREM 8. If $(G, Z)$ is free and $G$ is amenable, then $\pi$ is a homeomorphism.

Letting $(G, Z)$ be the transformation group of Theorem $3, G$ is amenable, and $(G, Z)$ is free, as otherwise there would be a finite, thus closed orbit. As $\pi$ is one-to-one, pr $\mathfrak{A}$ has only one point, and as $\pi^{\prime}$ is onto, $E T(\mathfrak{A})$ is uncountable.

\section{BibliograPHY} 321.

1. J. Dixmier, Algèbres quasi-unitaires, Comment. Math. Helv. 26 (1952), 275-

2. - Sur les $C^{*}$-algèbres, Bull. Soc. Math. France 88 (1960), 95-112.

3. —_ Les $C^{*}$-algèbres et leurs representations, Gauthier-Villars, Paris, 1964.

4. E. Effros, Structure in simplexes, Acta Math. (to appear).

5. H. Furstenberg, Strict ergodicity and transformations of the torus, Amer. J. Math. 83 (1961), 573-601.

6. J. Glimm, Families of induced representations, Pacific J. Math. 12 (1962), 885-911.

7. F. P. Greenleaf, Invariant means on topological groups and their applications, (to appear).

8. A. Guichardet, Charactères des algèbres de Banach involutives, Ann. Inst. Fourier (Grenoble) 13 (1962), 1-81.

9. J. von Neumann, On rings of operators. III, Ann. of Math. 41 (1940), 94-161. 1966.

10. R. Phelps, Lectures on Choquet's theorem, van Nostrand, Princeton, N. J.,

11. 1. E. Segal, $A$ class of operator algebras which are determined by groups, Duke Math. J. 18 (1951), 221-265.

12. E. Thoma, Über unitäre Darstellungen abzählbarer, diskreter Gruppen, Math. Ann. 153 (1964), 111-138.

\section{University of California at Berkeley, University of Pennsylvania AND YaLE UNIVERSITY}

